\title{
New Zealand national and regional nutrient mapping using the CLUES model
}

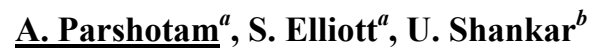 \\ ${ }^{a}$ National Institute of Water and Atmospheric Research (NIWA), Hamilton, New Zealand \\ ${ }^{b}$ National Institute of Water and Atmospheric Research (NIWA), Christchurch, New Zealand \\ Email: Aroon.Parshotam@niwa.co.nz
}

\begin{abstract}
CLUES is a GIS-based modelling system which assesses the effects of land use change on water quality and socio-economic indicators. It was developed by the National Institute of Water and Atmospheric Research (NIWA) in New Zealand and is an amalgamation of existing modelling and mapping procedures contributed by various New Zealand research organizations. CLUES allows users to create both land use and farm practice change scenarios (stocking rates, mitigation) using a range of tools. Results are available in map and tabular displays.
\end{abstract}

Our aim was to model the effects of land use change and intensification on nutrient loads and concentrations in streams in New Zealand, over recent periods of intensification (since 1996) and into the future (to 2020). The CLUES model was run for six scenarios reflecting changes in land use and stocking rates.

Predictions of the spatial distribution of nutrient loading to streams and changes over time are presented. The predicted total nitrogen $(\mathrm{N})$ load (the load entering streams) increased by $20.4 \%$ from 1996 to 2020 , reflecting both an increase in dairy area and intensification. Overall, $\mathrm{N}$ loads increased significantly over time, as a result of both intensification and land use change. Total phosphorous $(\mathrm{P})$ increased initially but decreased by a small amount overall due to the counterbalancing effects of changes in areas of dairying and changes in afforestation.

Regional analysis showed that $\mathrm{N}$ loads increased over time in all regions, except that in Auckland and Northland the load decreased slightly from 1996 to 2008, because the area of dairying decreased in those regions, and in Gisborne, N loads decreased from 1996 to 2020, during which time hill country sheep and beef areas decreased considerably and planted forest and scrub areas increased. The largest percentage increases occurred in the Canterbury and Southland regions, and were associated with the large increases in dairying.

P loads decreased only slightly overall nationally, with increases in some regions and decreases in others. In Northland, P load decreased because the area of dairying decreased and the area of forestry on vulnerable soils increased. In Northland, there was also a decrease in P, as sheep and beef areas reverted to scrub. In Bay of Plenty, there was a decrease in $\mathrm{P}$ due to the conversion of sheep and beef areas to forestry. In other regions, such as Gisborne and Hawke's Bay, there was an overall decrease in P load, because the effect of increased dairy was outweighed by the effect of afforestation. In areas such as Southland, though, there was an overall increase, due to increased dairying without afforestation, while in other regions such as Canterbury, P load increased due to intensification and increased dairy areas.

The overall implication from a modelling perspective is that land use change and intensification has and will continue to result in increased nutrient loadings and concentrations in New Zealand streams.

Keywords: $\quad$ GIS, nutrient mapping, CLUES, water quality, $N, P$ 


\section{INTRODUCTION}

Nutrient levels in New Zealand surface waters are low by international standards. However, there is great concern about current and potential eutrophication in lakes, and the impacts of intensification of pastoral agriculture. The CLUES (Catchment Land Use for Environmental Sustainability) project was initiated in 2003 by the Ministry of Agriculture and Forestry (MAF) and the Ministry for the Environment (MfE). The objective was to tie together a number of models into one GIS platform to facilitate assessments of the progressive effects of land use change on water quality in lakes, rivers and coastal regions (Elliott et al. 2008). The CLUES model has been used extensively in New Zealand to determine land-use change effects on water quality.

The purpose of the current work, commissioned by the Parliamentary Commissioner for the Environment (PCE), was to produce nutrient quality maps for New Zealand and to assess national and regional changes. A number of scenarios were assumed for the study and analysis.

Our aim was to model the effects of land use change and intensification on nutrient loads and concentrations in New Zealand streams over recent periods of intensification (since 1996) and into the future (to 2020). The CLUES model was run for six scenarios reflecting changes in land use and stocking rates. The stocking rates are effective stocking rates, that reflect increased farming intensity for a given land use type, representing changes in numbers of animals per unit area and increased production per animal. Both spatial distribution of nutrient loading to streams and changes over time are presented. Concentration model data are not presented in the current work.

\section{THE CLUES MODEL}

CLUES (Catchment Land Use for Environmental Sustainability) is a GIS-based model for predicting the effect of land use change and intensification on various socio-economic indicators, as well as on nutrient, $E$. Coli and sediment in surface water for each stream reach in New Zealand. The original development of CLUES was funded by MAF and MfE, and the model brings together various model components from NIWA, Landcare Research, AgResearch, and Plant and Food Research. The original model is described in Woods et al. (2006). References to modification to the model and various applications, along with other background material, are provided in the manual (Semadeni-Davies et al. 2011). The model has been set up with default input datasets and parameters, and is freely available from NIWA for download and use. The following predicted output variables for total nitrogen $(\mathrm{N})$ and total phosphorus $(\mathrm{P})$ were used in this study:

- Loads (tonnes). The mean annual load of TN or TP passing through the stream reach.

- Generated yield $\left(\mathrm{kg} \mathrm{ha}^{-1} \mathrm{yr}^{-1}\right)$. The mean annual load of nutrient generated in the catchment and entering the stream system (via surface or subsurface pathways) per unit area of land.

- Concentration $\left(\mathrm{mg} \mathrm{m}^{-3}\right)$. The predicted median concentration.

- Generated loads (tonnes). This is not a standard CLUES output, but was calculated from the subcatchment generated yields and areas, summed over the area of interest.

The key assumptions or features of the model most relevant to this project are:

- TP loading for pastoral land uses is determined from a simplified version of OVERSEER, as described in Woods et al. (2006). Default stocking rates are used for each region, based on the land use type and land slope. These default stocking rates are adjusted up or down depending on userentered relative stocking rates. The simplified OVERSEER model takes account of these stocking rates, along with the effects of slope, soil drainage class, rainfall, and soil type. Default fertiliser rates are used. This simplified model does not include 'farm-level' losses such as dairy effluent pond discharges.

- An additional source term for P is used for dairy areas, as described in Parshotam and Elliott (2009). This is a uniform value across New Zealand which is then modified by rainfall.

- An additional P source is applied to account for mass erosion, based on erosion rates which do not explicitly take account of land use effects. In this erosion model, sediment yields per area are determined as a function of geology and mean annual rainfall, but do not take land cover into account directly. In the CLUES P model, the sediment load is multiplied by a P concentration of sediment (see Woods et al. 2006) to derive the P load associated with erosion. This is a small term 
relative to the OVERSEER predicted $\mathrm{P}$ sources in pastoral areas, except where erosion rates are large.

- The assumptions for $\mathrm{N}$ are similar to those for $\mathrm{P}$, except that there is no erosion term, or additional source term for dairy.

- For both $\mathrm{N}$ and $\mathrm{P}$, the loading can be adjusted up or down to take account of mitigation measures. This feature was not used in the current study. Rather, it was assumed that mitigation measures remain at the current levels implicit in the calibration of the SPARROW component of CLUES.

Point sources, such as water treatment plants, freezing works and paper mills, are added, based on a survey of sources conducted around the year 2001. These remained fixed for past and future scenarios in this study. Urban areas have a diffuse source contribution, so that urban load increases as the extent of urbanization increases. In the standard CLUES model the country is subdivided into 10 subs-areas to reduce the datasets to a manageable size. Since this project required national runs, the CLUES model was set up and run for each region and the results collated. The results of the national CLUES runs were then mapped and summarised

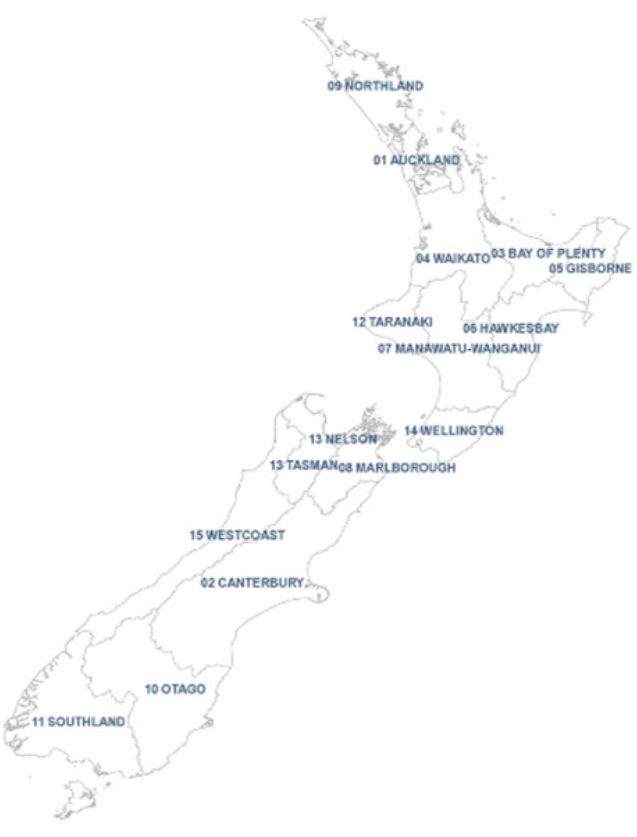

Figure 1. Regional boundaries and codes using standard GIS (ESRI ArcMap), database (MS-

Access) and spreadsheet (MS Excel) software. The results were collated by regional boundaries (not CLUES sub-areas) for reporting the results (see Figure 1 for the list of regions). The version of the CLUES model used was CLUES 10 for ArcGIS 10.

\section{CLUES INPUT DATA}

\subsection{Land use layers}

LURNZ (Land Use Rural New Zealand) land use grids were supplied by Motu Economic and Public Policy Research for the years 1996, 2008, and 2020, as described in Anastasiadis and Kerr (2013). Before these grids could be used in CLUES, the land use classes had to be re-classified to CLUES land use classes. The reclassified land use grids were then converted to polygon shape files for use in CLUES. For the year 2020, there is a \$5 carbon price and a \$25 carbon price land use scenario assumed in Motu's LURNZ (Land Use in Rural New Zealand) Climate Land Use Change Simulations. These land use scenarios reflect short term effects of carbon prices on land use in the NZ Emissions Trading System. Unless otherwise, specified, the $\$ 25$ carbon price was used as the 'default'.

\subsection{Stocking rates}

CLUES allows for stocking rates to be adjusted by a percentage of the default values, which are based on conditions in 2001. In this study, changes in stocking rates to represent changes in intensification (increase stock numbers or production per unit area) were

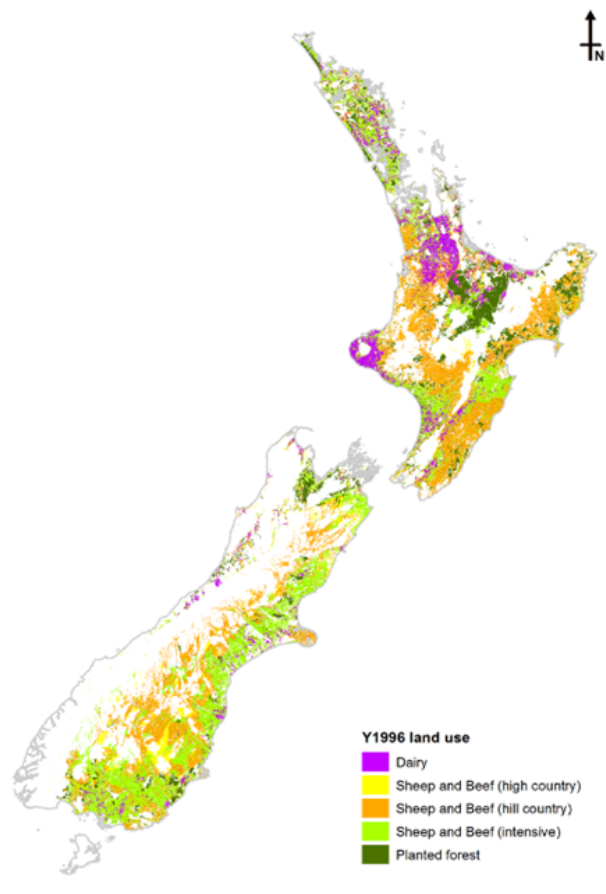

Figure 2. Year 1996 land use layer 
assessed by Motu (Anastasiadis and Kerr, 2013). Effective stocking rates were provided for each of 1996, 2008, and 2020, relative to (as a percentage of) 2001 values. For sheep and beef farming, a single relative value was provided nationally, whereas for dairy, regional values were used. These relative stocking rates were allocated to each CLUES sub-catchment based on the region code contained within CLUES (Woods et al. 2006). The stocking rates are expressed relative to 1996 (original data relative to 2001 is given by Anastasiadis and Kerr, 2013). In this work, the suffix S1 in a scenario refers to the 2008 stocking rates and the suffix S2 refers to 2020 stocking rates, respectively. The scenario Y2020 \$25 S2 therefore denotes the 2020 land use layer assumed, with a \$25 carbon price and with 2020 stocking rates.

\section{RESULTS AND DISCUSSION}

\subsection{Maps and breakdown of CLUES Land use layers}

Figure 2 presents a map of 1996 CLUES land use layers of interest for dairy, sheep and beef classes, and planted forest classes. Figure 3 presents past and future land use areas of interest. Note that this is an analysis of the input land use layer.
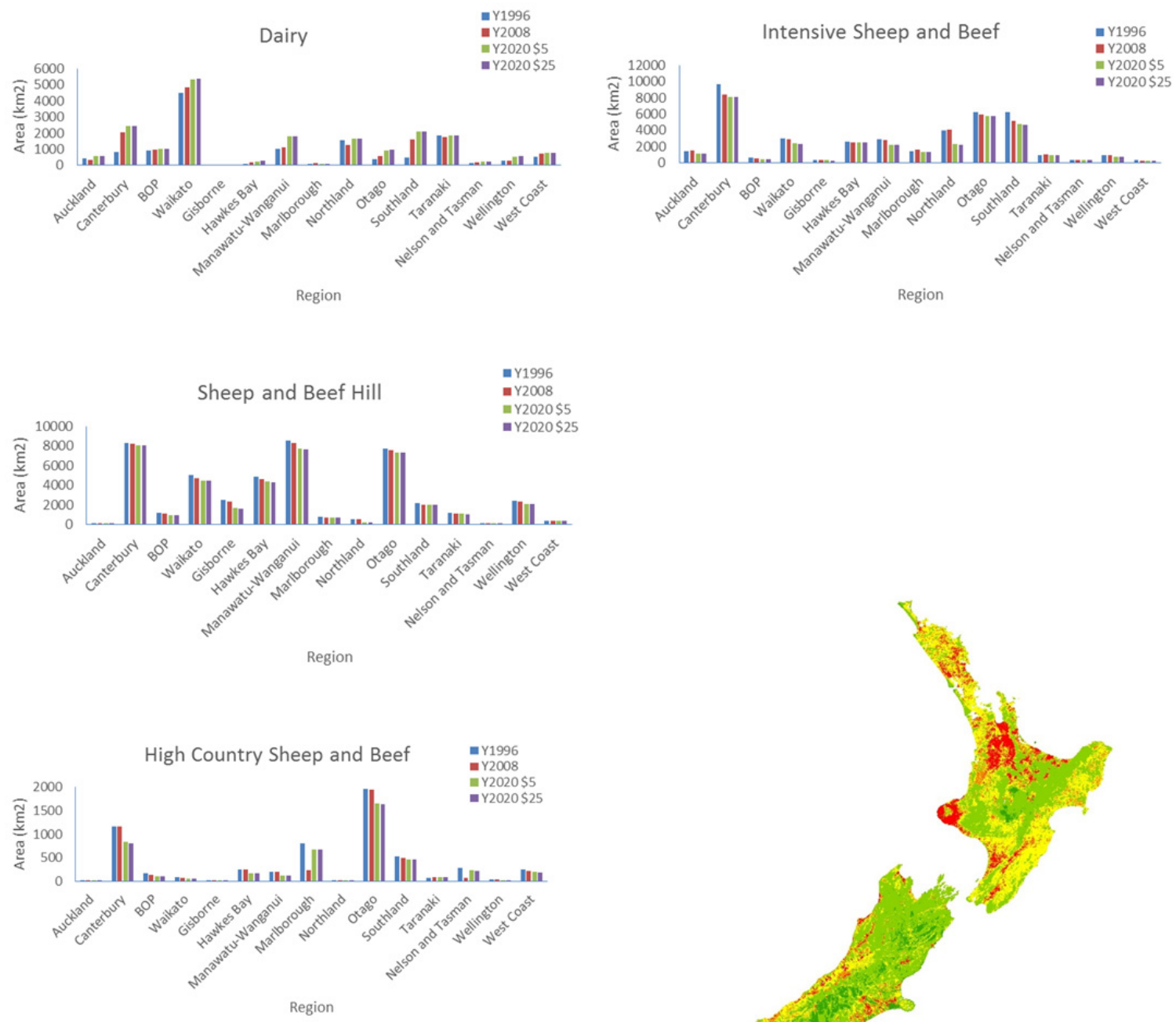

Figure 3. Key land use areas of interest

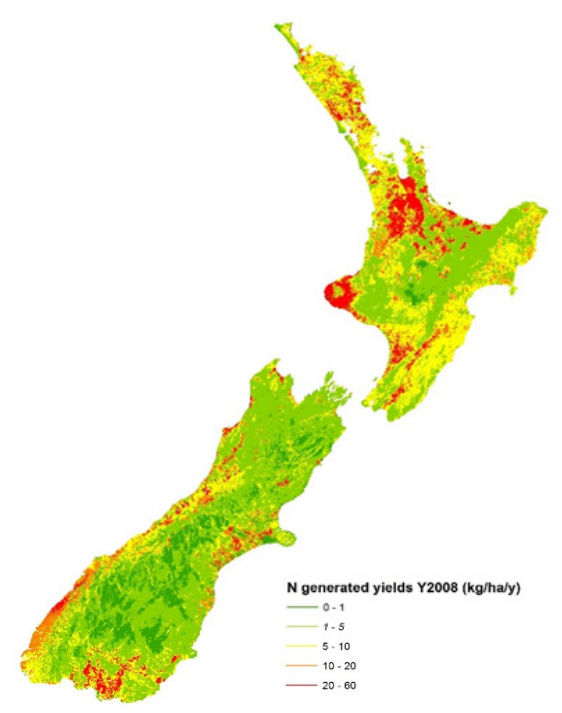

A $51 \%$ increase in the area of dairy farming nationally from 1996 to 2020 was predicted $\left(6653 \mathrm{~km}^{2}\right.$ increase

Figure 4. $\mathbf{N}$ generated yields for 2008 total, with $3779 \mathrm{~km}^{2}$ of that increase occurring from 2008 to $2020 \$ 25)$. The increase in dairy area was largely offset by a decrease in intensive sheep and beef (7862 $\mathrm{km}^{2}$ decrease from 1996 to 2020). The area of hill country sheep and beef decreased by $4989 \mathrm{~km}^{2}$ over that 
period, offset by an increase in the area of planted forest. The area of scrub decreased by $1348 \mathrm{~km}^{2}$, high country sheep and beef decreased by $1262 \mathrm{~km}^{2}$ and urban area increased by 115 $\mathrm{km}^{2}$. Regionally, the largest percentage increase (relative to 1996 value) in dairy area was in Gisborne (i.e., East Cape). The largest absolute increases in dairy area were in Canterbury $\left(2443 \mathrm{~km}^{2}\right)$, Waikato $\left(5371 \mathrm{~km}^{2}\right)$, Manawatu $\left(1808 \mathrm{~km}^{2}\right)$ and Southland $\left(2098 \mathrm{~km}^{2}\right)$. Increases in planted forest near or greater than 600 $\mathrm{km}^{2}$ occurred in Manawatu, Gisborne, Waikato and Hawke's Bay.

\subsection{Maps of $\mathbf{N}$ generated yields}

Maps of $\mathrm{N}$ generated yield predicted by the model (e.g. Figure 4 for 2008) show that large generated yields occurred in dairy farming areas. This is particularly evident in Bay of Plenty Southland,

Waikato, Taranaki and parts of Canterbury. As

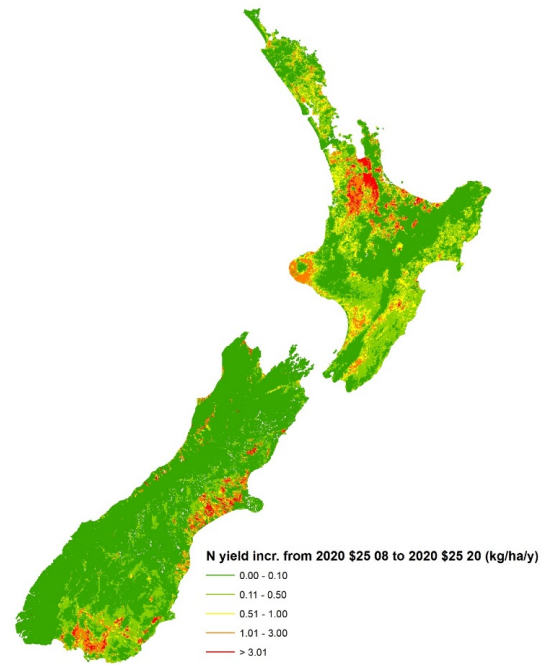

Figure 6. Increases in $\mathbf{N}$ generated yields due to intensification

Figure 5. $\mathrm{N}$ generated yield increases from 2008 to 2020.

dairying expands, the areas of large generated N yield increase (e.g., in Southland and Manawatu-Wanganui, see Figure 5).

There were large yield increases associated with point sources, but these don't show up in the map because they are associated only with the local catchment. There are some questionably large $\mathrm{N}$ yields simulated in the Fiordland area, which are the result of very high rainfall in conjunction with imperfect soil drainage. These $\mathrm{N}$ loads presumably would not result in high concentrations because they are discharged into very large volumes of water.

Maps of yield increases highlight the effect of new dairy areas. Some increases also occur in areas with fixed pastoral land use due to intensification. Yield decreases in some areas were due to afforestation, and because the land use model predicted that some dairy areas were converted to sheep and beef in the period from 1996 to 2008 .

Increases in $\mathrm{N}$ yields due to intensification from 2008 to 2020 are shown in Figure 6. The largest absolute increases occurred in dairying areas.

\subsection{Maps of $P$ generated yields}

The maps of $\mathrm{P}$ generated yield (Figure 7) show the largest $\mathrm{P}$ generated yields occurred in the Southern Alps. This is due to the large predicted erosion rates in that area. Large $\mathrm{P}$ generated yields also occurred in other areas with high rates of erosion (e.g., East Cape, parts of Northland). Large P yields also occurred in dairying areas such as Taranaki. Increases in $\mathrm{P}$ yield occurred in areas where dairying was introduced (Figure 8), such as Wairarapa, Manawatu and Southland. There are small predicted increases associated with

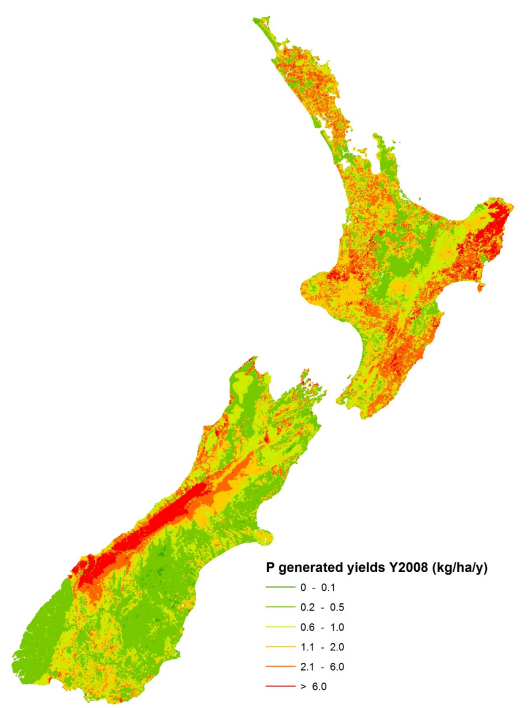


intensification (Figure 9), reflecting the small sensitivity of the OVERSEER P model used in CLUES to stocking rate (which is consistent with the full OVERSEER model). Decreases in P yield occurred in some areas, such as parts of East Cape (i.e., Gisborne and Bay of Plenty) which are associated with new areas of forestry. This reduction in yield occurred because the P loss associated with forestry is less than the P loss predicted by OVERSEER for pasture. However, these decreases were probably underestimated because the erosion component of $\mathrm{P}$ loss in the model does not change when land use is changed.

\subsection{National $\mathbf{N}$ and $P$ generated loads}

The predicted total generated loads of $\mathrm{N}$ entering streams across the country increased over time (see Table 1). Nationally, there was a slight increase from 1996 until 2008 and then a decrease in $\mathrm{P}$ load over time (data not shown). This was due to the counteracting effects of changes in areas of dairying and afforestation. Generally, and on analyzing loads and areas together, it may be shown that areas of sheep/beef decrease and go to either dairy (on flat land) or forestry or scrub (on hilly land). Resulting $\mathrm{N}$ increases are generally due to land use changes moving away from sheep and beef into dairy. Generally, P decreases or stays about the same because there is less erosion of $\mathrm{P}$ from hilly land going to scrub or forestry, counteracting the effect of increased dairying. The largest absolute increases in $\mathrm{N}$ and $\mathrm{P}$ occurred between 1996 and 2008 as rates of land use change (to dairy) were higher in most regions during this period. Assumed carbon prices made little difference: almost none for $\mathrm{N}$ and very small for $\mathrm{P}$.

Table 1. Summary of increases in predicted national $\mathrm{N}$ and $\mathrm{P}$ generated loads

\begin{tabular}{lcc}
\hline \multicolumn{1}{c}{ Scenario } & \multicolumn{2}{c}{ Increase from 1996 (\%) } \\
\cline { 2 - 3 } & $\begin{array}{c}\text { N generated } \\
\text { load }\end{array}$ & $\begin{array}{c}\text { P generated } \\
\text { load }\end{array}$ \\
\hline Y1996 & 0 & 0 \\
Y2008 & 8.9 & 0.36 \\
Y2020 \$5 S1 & 15.3 & -0.74 \\
Y2020 \$5 S2 & 20.2 & -0.55 \\
Y2020 \$25 S1 & 15.5 & -0.93 \\
Y2020 \$25 S2 & 20.4 & -0.74 \\
\hline
\end{tabular}

There was considerable regional variation in the changes (Figure 10). $\mathrm{N}$ load increased over time in all regions, with the exceptions of Auckland and Northland where the load decreased from 1996 to 2008 because the area of dairying decreased. In Gisborne, N loads decreased from 1996 to 2020, during which time hill country sheep and beef areas decreased considerably and planted forest and scrub areas increased.

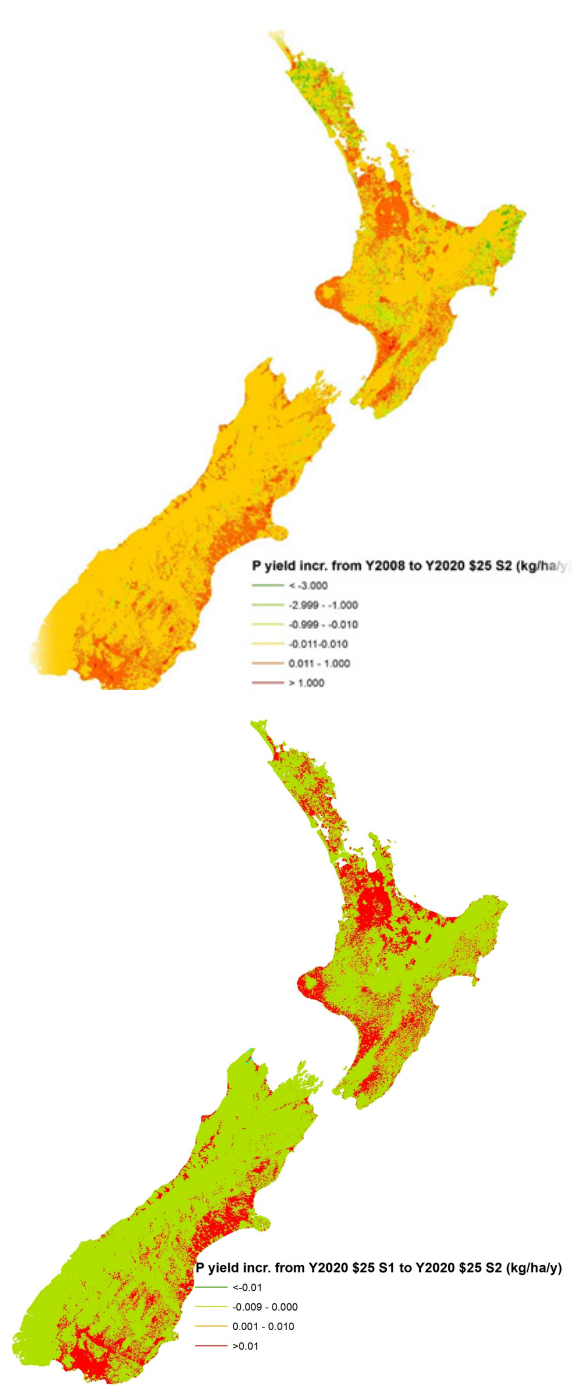

Figure 9. P generated yield increases due to intensification

In Auckland there was a prominent increase in $\mathrm{N}$ from a small base line over time. The largest percentage increase in $\mathrm{N}$ load occurred in Canterbury and Southland because there was a large percentage increase in dairying. In Waikato and Taranaki, there was a moderate increase in $\mathrm{N}$ with a medium drive from sheep and beef to dairy. In Otago, there was a large increase in $\mathrm{N}$ from a low baseline and in Nelson and Tasman there was a medium increase from a low baseline. Marlborough was a good example of there being no increase in nutrient yield as a result of no increase in dairying. In Gisborne, there was a decrease in $\mathrm{N}$ because areas of sheep and beef conversion into forestry and scrub. In Wellington, there was a large increase in $\mathrm{N}$ from a low base line, again driven by dairying. In Manawatu-Wanganui, there was a medium increase in $\mathrm{N}$ with a medium drive from sheep and beef to dairy. 
The predicted total generated loads of $\mathrm{P}$ entering streams did not change much over time (data not shown) but the change over time varied regionally (Figure 10).

In Northland, P load decreased because the area of dairying decreased and the area of forestry on vulnerable soils increased. In Northland, there was also a decrease in P because sheep and beef areas reverted to scrub. In Bay of Plenty, there was a decrease in P because sheep and beef areas were converted to forestry. In other regions such as Gisborne and Hawke's Bay there was an overall decrease because the effect of increased dairy was outweighed by the effect of afforestation. In areas such as Southland, though, there was an overall increase due to increased dairying rather than afforestation. The model probably underestimated the decrease in $\mathrm{P}$ from afforestation, because the erosion source term does not take account of land cover changes. It would be desirable to improve this aspect of the model in future.
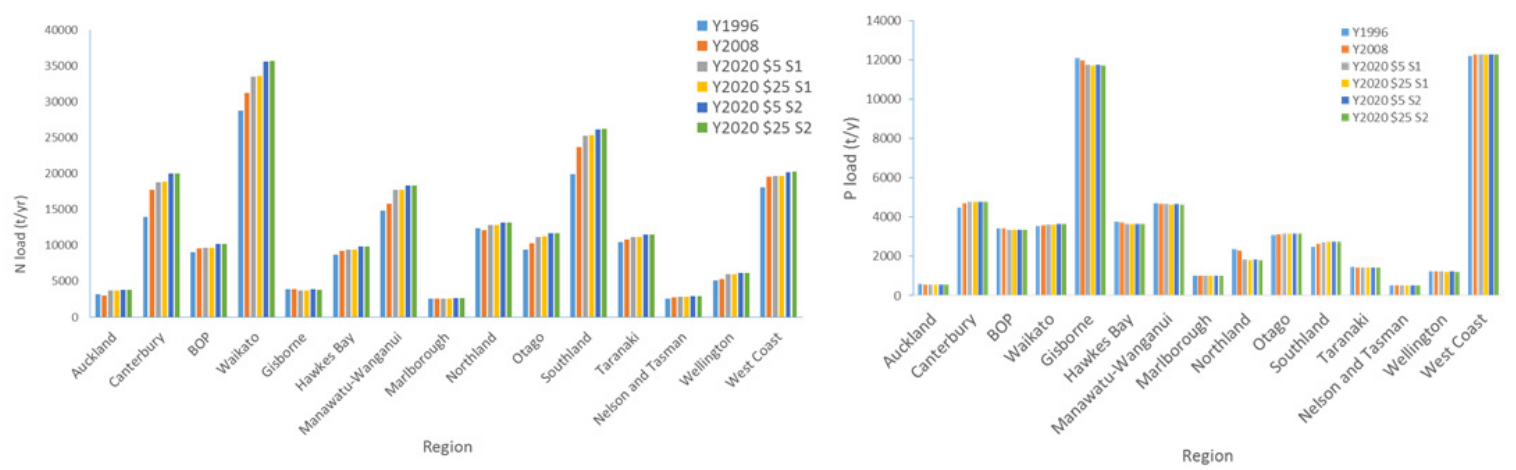

Figure 10. Regional N and P loads for various scenarios

\section{CONCLUSIONS}

The CLUES spatial decision support system is useful as a modelling tool for producing national and regional nutrient maps and model output data which may be further analyzed regionally and nationally to give an idea of land use effects on nutrient loadings. The overall implication from a modelling perspective is that land use change and intensification has and will continue to result in increased $\mathrm{N}$ and $\mathrm{P}$ loadings in New Zealand streams.

\section{ACKNOWLEDGMENTS}

The financial support from The Parliamentary Commissioner for the Environment including permission to present this work is gratefully acknowledged.

\section{REFERENCES}

Anastasiadis, S., Kerr, S. (2013) Landuse and Farming Intensity: For 1996, 2008 and 2020. Report for The Parliamentary Commissioner for the Environment. Motu Economic and Public Policy Research.

Elliott, S., McBride, G., Shankar, U., Semadeni-Davies, A., Quinn, J., Wheeler, D., Wedderburn, L., Small, B., Hewitt, A., Gibb, R., Parfitt, R., Clothier, B., Green, S., Harris, S., and Rys, G. (2008). CLUES Spatial DSS: From Farm-Scale Leaching Models to Regional Decision Support. International Congress on Environmental Modelling and Software Society (iEMSs), 2008.

Parshotam A. and Elliott, S. (2009). Application of CLUES to Five Monitored Dairy Catchments in New Zealand. NIWA Client report AGR08220 prepared for AgResearch.

Parshotam A., Elliott, S., Shankar, U., and Wadhwa, S. (2013). National nutrient mapping using the CLUES model, NIWA Client report for Parliamentary Commissioner for the Environment, HAM2013-086. Project MCT13202.

Semadeni-Davies, A., Elliott, S., Shankar, U. (2011) The CLUES Project: Tutorial Manual for CLUES 3.1. Prepared for Ministry of Agriculture and Forestry. NIWA Client Report HAM2011-003.

Woods, R., Elliott, S., Shankar, U., Bidwell, V., Harris, S., Wheeler, D., Clothier, B., Green, S., Hewitt, A., Gibb, R., Parfitt, R. (2006) The CLUES Project: Predicting the Effects of Land-use on Water Quality Stage II. NIWA Client Report HAM2006-096. National Institute of Water and Atmospheric Research. Hamilton, New Zealand. 